\title{
THE GHOST OF A DWARF GALAXY: FOSSILS OF THE HIERARCHICAL FORMATION OF THE NEARBY SPIRAL GALAXY NGC 5907
}

\author{
David Martínez-Delgado, ${ }^{1,2,3}$ Jorge Peñarrubia, ${ }^{4}$ R. Jay Gabany, ${ }^{5}$ Ignacio Trujillo, ${ }^{1,3}$ \\ Steven R. Majewski, ${ }^{6}$ and M. Pohlen ${ }^{7}$ \\ Received 2008 May 7; accepted 2008 August 12
}

\begin{abstract}
We present an extragalactic perspective of an extended stellar tidal stream wrapping around the edge-on spiral galaxy NGC 5907. Our deep images reveal for the first time a large-scale complex of arcing loops that is an excellent example of how low-mass satellite accretion can produce an interwoven, rosette-like structure of debris dispersed in the halo of its host galaxy. The existence of this structure, which has probably formed and survived for several gigayears, confirms that halos of spiral galaxies in the local universe may still contain a significant number of galactic fossils from their hierarchical formation. To examine the validity of the external accretion scenario, we present $N$-body simulations of the tidal disruption of a dwarf galaxy-like system in a disk galaxy plus dark halo potential that demonstrate that most of the tidal features observed in NGC 5907 can be explained by a single accretion event. Unfortunately, with no kinematic data and only the projected geometry of the stream as a constraint, the parameters of our model are considerably degenerate and, for now, must be considered illustrative only. Interestingly, NGC 5907 has long been considered a prototypical example of a warped spiral in relative isolation. The presence of an extended tidal stream challenges this picture and suggests that the gravitational perturbations induced by the stream progenitor must be considered as a possible cause for the warp. The detection of an old, complex tidal stream in a nearby galaxy with rather modest instrumentation points to the viability of surveys to find extragalactic tidal substructures around spiral galaxies in the Local Volume $(<15 \mathrm{Mpc})$, with the prospect of obtaining a census with enough statistical significance to be compared with cosmological simulations.
\end{abstract}

Subject headings: dark matter — galaxies: dwarf — galaxies: evolution — galaxies: halos — galaxies: individual (NGC 5907) — galaxies: interactions

Online material: color figure, mpeg animation

\section{INTRODUCTION}

The cold dark matter $(\Lambda \mathrm{CDM})$ paradigm predicts that stellar halos surrounding large spiral galaxies formed through the accretion and tidal disruption of satellite galaxies, a notion previously postulated (Searle \& Zinn 1978) on empirical grounds from the character of stellar populations found in our own Milky Way halo. Simulations have shown that the fossil records of those merger events may be detected nowadays in the form of coherent stellar structures in the outer regions of the host galaxies. The most spectacular of these fossil structures is probably the formation of dynamically cold stellar streams, which are similar to long rivers of stars (and likely dark matter; Peñarrubia et al. 2008b) tidally stripped from a disrupting dwarf galaxy and wrapped around the host galaxy disk, roughly tracing the orbit of the progenitor satellite. The now well-studied Sagittarius (Sgr) tidal stream surrounding the Milky Way (Ibata et al. 2001b; Majewski et al. 2003; Martínez-Delgado et al. 2004) and the giant stream in the Andromeda galaxy (Ibata et al. 2001a) are archetypes in the Local Group of these kinds of fossilized satellite galaxy mergers and provide obvious support for the scenario that tidally disrupted dwarf galaxies are an important contributor to the stellar halos of disk galaxies.

\footnotetext{
${ }^{1}$ Instituto de Astrofísica de Canarias, 38200 La Laguna, Tenerife, Spain.

2 Max-Planck-Institut für Astronomie, Königstuhl 17, D-69117 Heidelberg, Germany.

3 Ramón y Cajal Fellow.

${ }^{4}$ University of Victoria, Victoria, BC V8W 2Y2, Canada.

5 Blackbird Observatory, Cloudcroft, NM 88317.

${ }^{6}$ Department of Astronomy, University of Virginia, Charlottesville, VA 22904.

${ }^{7}$ Cardiff University, School of Physics and Astronomy, Cardiff CF24 3AA, UK.
}

Unfortunately, while the Milky Way and even M31 streams are close enough to be resolved into individual stars that can be studied in detail, these nearby streams can also span vast areas of sky, which currently presents particular challenges to their study. In contrast, tidal streams around more distant systems can be imaged in commensurately smaller fields of view and - at least for those streams seen in diffuse light - with equal contrast, given the constancy of surface brightness with distance. However, over the past decade, only a few cases of confirmed stellar tidal streams have been detected in disk galaxies outside the Local Group (e.g., Malin \& Hadley 1997; Forbes et al. 2003; Pohlen et al. 2004; and references therein), in contrast with the increasing number of tidal structures detected in the Milky Way and M31 in recent years. The presence of a multitude of tidal streams, arcs, shells, and other irregular structures possibly related to ancient merger events in the deep panoramic view of the Andromeda halo (Ibata et al. 2007) reveals the level of stellar substructure that might be present in the halos of nearby external spiral galaxies.

Extragalactic streams also offer a unique perspective to address some interesting questions: Is the abundant number of stellar streams exceptional in the Local Group, or are tidal streams as common as has been predicted by current cosmological models? What can we learn about the dark matter halos of the host galaxies from these streams? Can we use these systems to study the star formation history of the disrupting dwarf galaxies? The frequency of streams and their stellar population and orbital properties provide important clues to the nature of the stream progenitors, lend insights into the underlying gravitational potential and structure of the massive dark halo they inhabit, and constrain hierarchical models of galaxy formation favored by current cosmological models. In that respect, an interesting parameter that may possibly be 
constrained through the detection of tidal streams in external galaxies is the flattening of the dark matter halos surrounding the host galaxies. The spatial and kinematic distributions of stream particles are fairly sensitive to this quantity (e.g., Johnston et al. 2002, 2005; Helmi 2004; Law et al. 2005; Peñarrubia et al. 2006; and references therein), and the shapes and orientations of dark matter halos with respect to the disk axisymmetry plane reflect the nature of dark matter (see, e.g., Dubinsky \& Calberg [1991], Yoshida et al. [2000], and Davé et al. [2001] for shape estimates for cold, self-interacting, and hot dark matter models, respectively). For example, $\Lambda \mathrm{CDM}$ models predict that dark matter halos are triaxial, with minor axes perpendicular to the disk plane. On average, the minor-to-major axis ratio near the center is $c / a \simeq 0.7$ (Hayashi et al. 2007), although halos may become considerably rounder after baryonic galactic components have formed (Kazantzidis et al. 2004) or if dark matter is warm (Biermann \& Munyaneza 2007). Thus, using tidal streams in external galaxies might be an ideal tool to obtain a significant statistical sample of flattening values that can be compared against predictions from cosmological models.

These arguments suggest that a systematic survey for tidal streams around extragalactic systems would provide a new way to explore galaxy structure and evolution, with direct constraints on cosmological models. Undertaking such a tidal stream survey was urged by Johnston et al. (2001) in their comprehensive discussion of the appearance and detectability of extragalactic tidal streams. Their analysis suggests that a survey of 100 parent galaxies reaching a surface brightness of $30 \mathrm{mag} \operatorname{arcsec}^{-2}$ would reveal many tens of tidal features; a slightly higher estimate of about one per galaxy is obtained in their most recent reanalysis of the problem (Bullock \& Johnston 2005). These authors also point out the advantage of exploring thin, edge-on disk systems because of the favorable orientation for detecting low surface brightness features against the light of the parent system and the ability to assess limits on the mass ratio of the satellite and parent galaxy by whether the disk of the parent appears to be disturbed.

Johnston et al. (2001) estimated that a search strategy designed to obtain reliable photometry along the stream length would require about 10 times longer exposure times that those aimed at detection only. We adopted this approach of striving for deep images to detect the full path of the tidal stream, which, in the absence of kinematic data, is the main input for comparison with $N$-body simulations (see $\S 4$ ). Using this strategy, we started a pilot survey on some nearby galaxies which has proven the concept with the discovery of a giant stellar tidal stream around the spiral galaxy NGC 4013 (Martínez-Delgado et al. 2008) and the detection of the faintest parts of the extended disk of the nearby spiral galaxy M94 (I. Trujillo et al. 2008, in preparation).

With the hope of improving our understanding of the known tidal stream in NGC 5907, discovered by Shang et al. (1998), and to demonstrate the sensitivity of the small-aperture telescope used in our survey (see $\S 2.2$ ), we reobserved this system as a commissioning target. In this paper we present our very deep image of NGC 5907 that reveals additional loops of debris in the halo (see $\S 3.1$ ) beyond those reported by Shang et al. (1998). The $N$-body simulations, described in $\S 4$, show that most of the newly found features can be explained as pieces of a spectacular, multiply wrapped stream of debris from the tidal disruption of a single companion galaxy. We also discuss in $\S 5$ the applications of such surveys to glean a deeper theoretical understanding of galaxy formation and evolution, beyond placing strong constraints on present cosmological scenarios. This foray into a more systematic look for tidal streams in the nearby universe has yielded promising results that encourage more aggressive attention to this new way of understanding galaxy structure and evolution as advocated by the above theoretical studies.

\section{TARGET, OBSERVATIONS, AND DATA REDUCTION}

\subsection{The Target: NGC 5907}

NGC 5907 is a nearby (14 Mpc; Zepf et al. 2000), edge-on Sc galaxy, very similar (e.g., type, absolute magnitude, and rotation speed) to the Milky Way. NGC 5907 has frequently been the target of deep surveys (radio, optical, and near-infrared) because it is an apparently undisturbed, almost edge-on disk useful for exploring the properties of spiral disks, determining the properties of dust and gas in disk galaxies, testing for the prevalence of luminous thick disks and halos, searching for warps, and exploring the strength of dark matter in disks (e.g., Sancisi 1976; van der Kruit \& Searle 1981; Casertano 1983; Skrutskie et al. 1985; Sasaki 1987; Barnaby \& Thronson 1992; Morrison et al. 1994; Sackett et al. 1994; Lequeux et al. 1996, 1998; Rand 1996; Dumke et al. 1997; Rudy et al. 1997; James \& Casali 1998; Xilouris et al. 1999; Zheng et al. 1999). Although VLA H i maps of this galaxy show that it has a large-scale warped gas disk (Sancisi 1976), NGC 5907 was long considered to be a prototypical example of a warped disk galaxy existing in relative isolation, since the identification of a neighboring, interacting galaxy was lacking. However, deep imaging of NGC 5907 by Shang et al. (1998) has revealed a very faint, elliptically shaped ring around the disk, and this has been interpreted to be the remnant of a tidally disrupted dwarf satellite galaxy, an analog to the debris stream created by the Milky WaySgr interaction, although at higher surface brightness.

\subsection{Observation and Data Reduction}

We have obtained deep optical images of NGC 5907 with the $0.5 \mathrm{~m}$ Ritchey-Chrétien telescope of the BlackBird Remote Observatory (BBRO), situated in the Sacramento Mountains (New Mexico). We used a Santa Barbara Instrument Group (SBIG) STL-11100 CCD camera, which yields a large field of view $\left(27.7^{\prime} \times 18.2^{\prime}\right)$ at a plate scale of $0.45^{\prime \prime} \mathrm{pixel}^{-1}$. The image set consists of multiple deep exposures with a noninfrared, clear luminance $(3500<\lambda<8500)$ and red, green, and blue filters from the SBIG Custom Scientific filter set. Table 1 provides a summary of the images collected during different dark sky observing runs during the period 2006 June through August. The third column refers to the total exposure time of the co-added images from each filter obtained with each run.

The science images were reduced using the standard procedures for bias correction and flat-fielding. A master dark and bias frame was created by combining 10 dark subexposures, each produced at the same exposure length and camera temperature settings used for the luminance and the filtered images. A master flat was produced by combining 10 separate sky flat exposures for each filter. The red, green, and blue filtered exposures were separately combined (using a median procedure) to produce red, green, and blue master images that represented the total exposure time for each color filter. These color images were obtained to investigate the color of the structures surrounding the galaxy and to extend the overall exposure of the final luminance image. The master images for each filter were subsequently summed to produce a synthetic luminance image that represented the total exposure time of all the color filter data. The synthetic luminance image was then combined (using a median procedure) with all the clear filtered luminance subexposures (see Table 1) to increase the contrast of the diffuse light. The resulting final image thus represents the sum of all available CCD exposures collected for this project, with an accumulated exposure time of $11.35 \mathrm{hr}$ (including $5.75 \mathrm{hr}$ in the clear luminance filter). 
TABLE 1

JoURNAL OF OBSERVATIONS

\begin{tabular}{|c|c|c|}
\hline Date & Filter & $\begin{array}{c}\text { Total Exposure Time } \\
\text { (s) }\end{array}$ \\
\hline 2006 Jun 6 ....................... & Blue & 6480 \\
\hline 2006 Jun 6 .................... & Clear luminance & 9000 \\
\hline 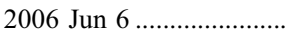 & Green & 1080 \\
\hline 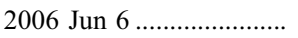 & Red & 5400 \\
\hline 2006 Jun 7 ...................... & Clear luminance & 3600 \\
\hline 2006 Jun 7 ..................... & Green & 2160 \\
\hline 2006 Jun 9 ....................... & Clear luminance & 5400 \\
\hline 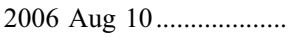 & Blue & 2160 \\
\hline 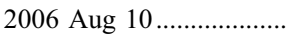 & Clear luminance & 2700 \\
\hline 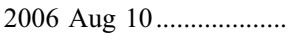 & Green & 1080 \\
\hline 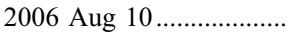 & Red & 1800 \\
\hline
\end{tabular}

To enhance the faint structures around NGC 5907 and disentangle their true appearance from image noise effects, the contrast and detail of the summed image were optimized using two wellknown image processing techniques: (1) a histogram equalization of the image using a nonlinear transfer function ${ }^{8}$ and (2) Gaussian blur filtering (Davies 1990, p. 42; Haralick \& Shapiro 1992). The first method is very effective at revealing very faint features in images that contain a large dynamical range, as in the case of a spiral galaxy with a very bright, compact stellar disk surrounded by a very faint tidal stream. With the purpose of suppressing the bright portions of the image and intensifying the fainter parts of the stream, histogram equalization employs a monotonic, nonlinear mapping which reassigns the intensity values of pixels in the input image such that the output image contains a uniform distribution of intensities. In our case, we used an iterative process that involves several passes of an S-shaped histogram operator to the total image. While this method was successful in revealing the presence of the surrounding faint structure, it also resulted in the oversaturation of contrast and significantly reduced intensity variances within the stellar stream. Once the image was contrasted, a low-pass Gaussian filter with a radius of 2 pixels was applied to reduce the low-level noise contaminating the image. The resulting noise-filtered image is shown in Figure 1.

As discussed above, our search technique was designed to very clearly reveal the position and morphology of faint structures (e.g., by combining all the available images obtained in different filters with the clear luminance deep images) but does not permit accurate photometry. Therefore, follow-up photometric observations are needed to measure accurate surface brightnesses and to estimate the total luminosity of the detected tidal debris (see $\S 3.3$ ). Furthermore, the use of an uncalibrated luminance filter prevents us from estimating the surface brightness limit that we reached in our deep probe of the NGC 5907 halo in any particular standard photometric band. However, the detection of obvious diffuse light structures fainter than those reported in previous studies (with a surface brightness as faint as $\Sigma_{R} \sim 27.7$ mag $\operatorname{arcsec}^{-2}$; see $\S 3.3$ ) suggests that our images reach a surface brightness magnitude fainter than the $R=27$ mag $\operatorname{arcsec}^{-2}$ limit of the deep NGC 5907 image by Morrison et al. (1994) and Sackett et al. (1994) and the $R=28.7 \mathrm{mag} \mathrm{arcsec}^{-2}$ limited image by Zheng et al. (1999). Regardless, this result is a striking example of the scientific potential of modest-aperture telescopes $(0.5 \mathrm{~m})$ operating under very dark skies for probing the diffuse structure of galaxies.

\footnotetext{
${ }^{8}$ See http://homepages.inf.ed.ac.uk/rbf/HIPR2/histeq.htm and references therein
}

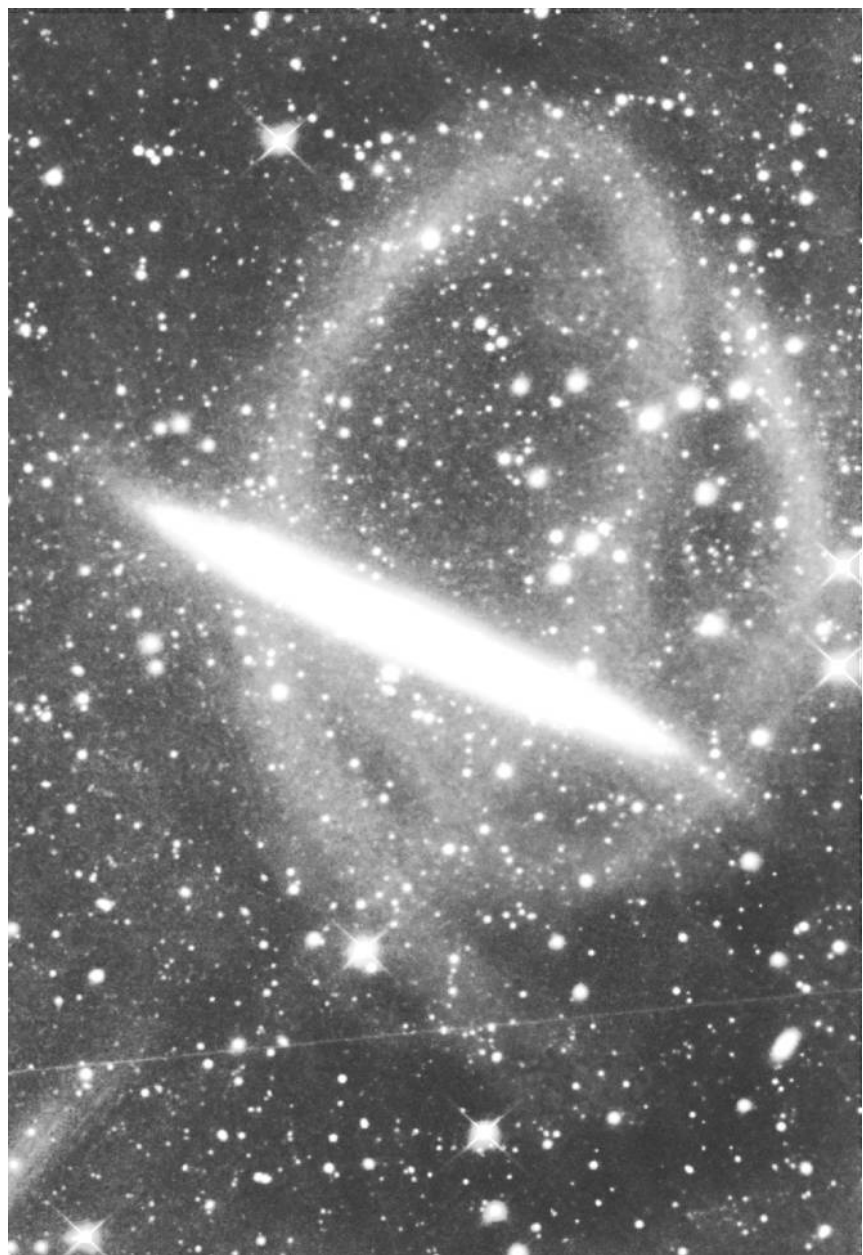

FIG. 1.- Image of NGC 5907 obtained with the BBRO $0.5 \mathrm{~m}$ telescope. The total exposure time of this image is $11.35 \mathrm{hr}$, co-adding all images obtained in this project (see Table 1). The image has dimensions of $18.2^{\prime} \times 27.7^{\prime}$, which, at the distance of NGC 5907 , is $\sim 75 \times 115 \mathrm{kpc}$. For a better comparison with the $N$-body simulations given in Fig. 4, this image is shown with east up and north to the right. The linear diagonal feature in the lower left corner of the image is spurious light from deflection of a bright star off the edge of the CCD chip. The faint halos surrounding the field stars are due to the pass of the Gaussian filter mentioned in $\S 2.2$.

\section{THE TIDAL STREAM(S) OF NGC 5907}

\subsection{Observed Morphology of the NGC 5907 System}

\subsubsection{Truncation and Optical Warp}

In this section we focus on the outer, diffuse light properties revealed in Figures 1 and 2, but several comments about the disk are worth pointing out briefly. Our image shows that the disk of NGC 5907 extends in diameter well beyond where most previous images have tracked its disk light. The existence of this light challenges previous claims that the disk is sharply truncated (in the sense of the galaxy's outer edge) at a nominal cutoff radius of about $6^{\prime}$ from the center (van der Kruit \& Searle 1981; Sasaki 1987; Morrison et al. 1994). From the original radial profiles (van der Kruit \& Searle 1981 and Sasaki 1987; Figs. 9 and 7, respectively) there is clearly a radial break feature visible; however, it would be better described as a sharp change of slope rather than a complete cutoff. This profile shape, well fitted with a broken exponential function, is a relatively common feature of late-type spiral galaxies (Pohlen \& Trujillo 2006; Erwin et al. 2008).

Figures 1 and 2 clearly show that NGC 5907 has a stellar warp, which clearly extends after the nominal cutoff radius. The observable faint radial extensions of the disk are strongly bent in the 


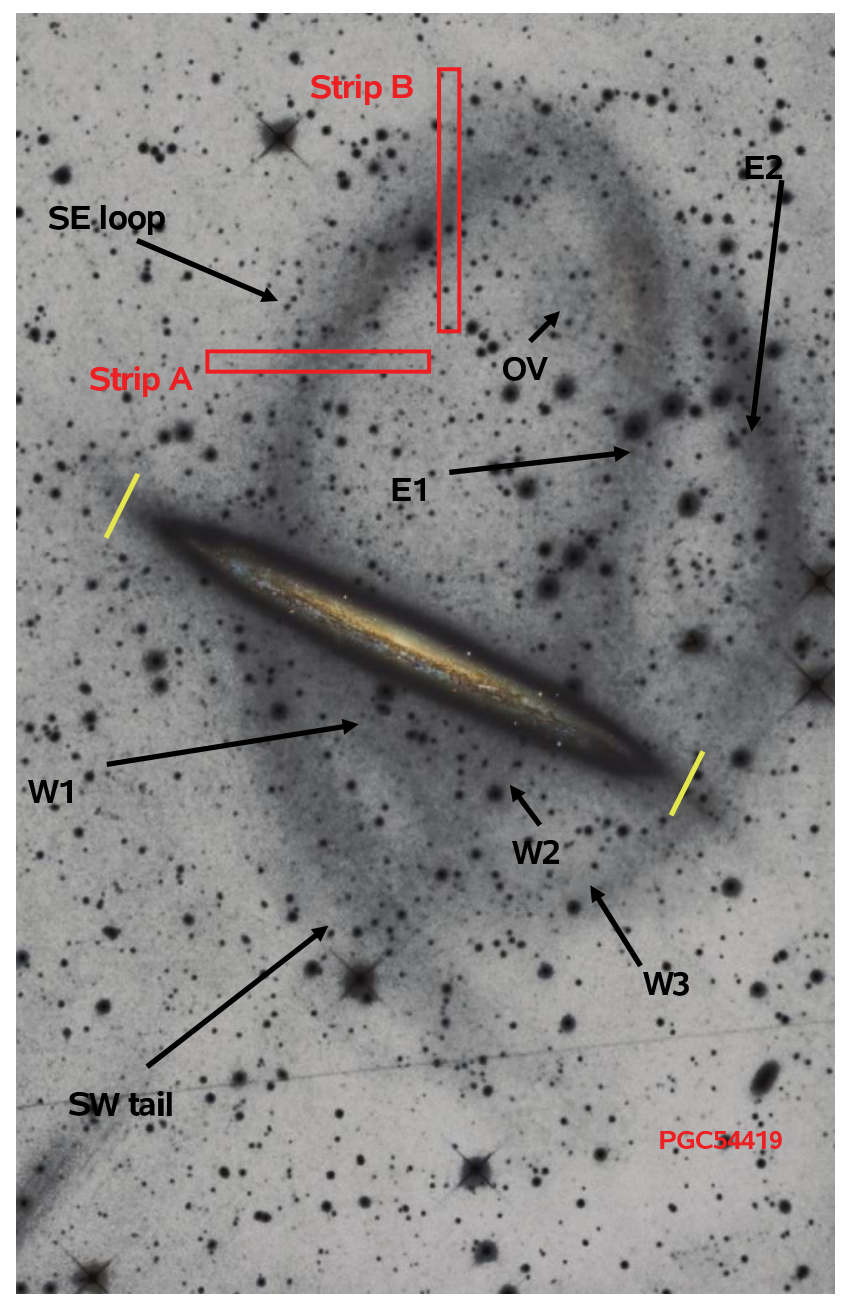

FIG. 2.-Identified photometric features detected in the image of NGC 5907 obtained with the BBRO $0.5 \mathrm{~m}$ telescope and discussed in $\S 3.1$. For reference, a color image obtained with the same telescope (see Table 1) has been superimposed on the saturated disk region of the galaxy. The positions of the two different strips used to measure the width of the stream $(\operatorname{see} \S 3.2)$ are also indicated by red rectangles. The yellow lines mark the position of the nominal cutoff radius (see $\S 2.2$ ). For comparison purposes, north is to the right and east is up.

same sense as the obvious H I warp found by Sancisi (1976) and are most strongly visible on the northwest disk edge. Note that these same extended disk features can also be seen in Figure 2 of Shang et al. (1998) and almost in the near-infrared image of Barnaby \& Thronson (1992). Evidence for the warp in optical light was also previously seen in the optical disk at smaller radii by Sasaki (1987), Florido et al. (1992), and Morrison et al. (1994) and was, of course, also pointed out by Shang et al. (1998).

\subsubsection{Diffuse Light Structures}

The most striking feature, and the primary focus of the present discussion, is the newly revealed network of arcing loops of diffuse light around NGC 5907 that are likely the product of the classical satellite tidal disruption process. Our deep image (see Fig. 2) reveals that the ring-shaped feature to the southeast-we refer to this here as the SE loop-discovered by Shang et al. (1998) is only the brighter part of a large-scale complex of stellar debris arcs around NGC 5907. We count about a half-dozen other additional arclike features in our image. On the eastern side, it is possible to detect the full extension of the SE loop, turning around and falling toward the disk. This yields a vertical structure (labeled
E1 in Fig. 2) that crosses the galactic plane and possibly extends to the other side of the galaxy. In addition, an independent structure (labeled E2), somewhat parallel to the SE loop, can be seen to be shifted about $\sim 10^{\prime}$ to the northwest of the Shang et al. (1998) structure and sweeping almost $180^{\circ}$ around the center of NGC 5907.

Although the accretion origin of this structure appears to be a reasonable hypothesis, we must point out its extraordinary complexity, especially on the western side of NGC 5907, where we find a faint, long tail extending $\sim 25 \mathrm{kpc}$ from the disk, labeled SW tail in Figure 2 and previously reported only in some studies (see Fig. $1 b$ in Reshetnikov \& Sotnikova 2000). From south to north, three additional arcs are detected on the west side of the disk (labeled W1, W2, and W3). These features appear to cross each other fairly closely to the disk major axis of NGC 5907, which makes it difficult to discern which of these arcs is the continuation of the features observed on the eastern side, that is, the SE loop, E1, and E2.

For example, it is obvious that the SE loop extends to the western side, but it becomes unclear which path it takes (e.g., SW tail or W1). On the other hand, it is clear that the W3 arc is the westernmost continuation of the E2 loop. Interestingly, this stream piece turns around, crosses the W2 arc, and finishes in the main disk again, forming the $\mathrm{W} 1$ arc. It is, however, more difficult to assess whether the W2 arc (the most diffuse discernible structure, highly contaminated by the diffuse light of the galaxy disk) or $\mathrm{W} 1$ could be the western extension of the E1 tail.

\subsubsection{Evidence for a Single Coherent Structure}

With the image in Figure 2 alone it is impossible to conclude whether the smaller features on the western side are the continuation of the loops on the eastern side, and thus whether the whole set of these identified tidal structures originates from a single or different accretion events. In general, the largest features (e.g., the SE and E2 loops) have similar shapes, angular lengths, radii of curvature, and separations from the center of NGC 5907. In addition, all of the features (large and small) share a similar projected width, $\sim 7 \mathrm{kpc}$ (consistent with the value reported by Shang et al. 1998; see $\S 3.3$ ), as well as a similar, rather bright surface brightness (see $\S 3.3$ ). These similarities all provide circumstantial evidence that the various features may all come from a single, old $^{9}$ stellar tidal stream, similar to the multiply wrapped Sgr dwarf galaxy stream around the Milky Way. These structures provide a rather unique and spectacular external view of the classical rosettelike, multiloop patterns expected for tidally disrupting dwarf galaxies seen in $N$-body simulations (such as those made of the Sgr system; e.g., Johnston et al. 1995; Ibata \& Lewis 1998; MartínezDelgado et al. 2004; Law et al. 2005). There are further analogies of the NGC 5907 structures with the Sgr stream in that they have a similar linear cross-sectional width (suggesting similarly massed progenitors), apogalacticon and apparent orbital ellipticity (suggesting a similar orbit), and surface brightness and length (suggesting a similar mass-loss rate and age of interaction). In $\S 4$ we explore this working hypothesis in detail and provide a comprehensive, illustrative model that further supports the notion that a single, Sgr-like system could create a substantial fraction of the newly found diffuse structures.

Interestingly, while the surface brightness of these loops does show some modulation, nowhere among these features do we see a bright spot that could be identified with a remaining dwarf

\footnotetext{
${ }^{9}$ Following Johnston et al. (2001), the age of the trail here refers to the epoch when the debris was unbound from the satellite core (see Fig. 4) and not to the age of its stellar population, which is unknown.
} 
satellite core. Thus, our image provides no definitive insights into the current position and final fate (i.e., whether or not it is completely disrupted by now) of the progenitor satellite galaxy (since it could be hidden behind the disk). However, the full panoramic view of the NGC 5907 system in Figure 1 provides no evidence for any association of the stellar streams with the dwarf irregular galaxy PGC 54419 (a confirmed satellite of NGC 5907 situated at $36.9 \mathrm{kpc}$ projected distance from its center), as proposed by Shang et al. (1998). We return to this subject in $\S 5$.

\subsection{Diffuse Light: Halo or Stream?}

As mentioned above, there have been a number of deep photometric studies of NGC 5907, and a popular point of discussion is the claimed detection and properties of "excess," nondisk light away from and perpendicular to the disk and commonly attributed to a diffuse luminous halo (Sackett et al. 1994; Lequeux et al. 1996, 1998; Rudy et al. 1997; James \& Casali 1998; Zepf et al. 2000; Irwin \& Madden 2006). Figure 1 clearly shows how these previous studies may have been affected by the coherent tidal stream structures, especially W1 and W2, which lie along the southwestern minor axis at distances where such studies have made the "halo" detections. Indeed, the very shape of the "inner triangle" of light formed by W1 and W2 can be discerned in the image analyzed by Lequeux et al. (1996; see, e.g., their Fig. 1), and a similar asymmetry seen in the photometric data by Sackett et al. (1994) may also be explained by these same structures (e.g., see the bottom right panel of Fig. 2 in Sackett et al. 1994).

One wonders whether the presence of these structures may also explain the differences in the colors of the halo light found on either side of the disk shown in Figure 1 of Lequeux et al. (1998). Zheng et al. (1999) have previously suspected that the presence of tidal debris light (particularly on the western side) might significantly contaminate the halo light that was previously reported; these authors concluded that, when combined with residual scattered light from foreground stars, this debris effectively reduces the need for a faint, extended halo. We concur that indeed these previous studies were probably strongly affected by the presence of the $\mathrm{W}$ features; but, of course, this is actually halo light, it is just in the form of tidal debris in what is obviously the active hierarchical formation of the NGC 5907 halo. However, a low surface brightness halo $\left(\Sigma_{R} \sim 30-31 \mathrm{mag} \operatorname{arcsec}^{-2}\right)$ similar to M31 could be present (J. Fliri et al. 2008, in preparation).

\subsection{Estimating the Surface Brightness, Total Luminosity, and Stellar Mass of the NGC 5907 Tidal Stream}

Ideally, one would like to use the BBRO image to measure the surface brightness of the observed stream. However, there are some concerns that prevent us from extracting this information directly from this image. First of all, the image is the result of the combination of multiple images in different filters with those taken using an extremely wide (luminance) filter to maximize the photon collection. This makes a calibration of the stars present in the BBRO image using the same stars observed through standard filters nontrivial. Significant color corrections are expected.

In addition, the resulting background on the BBRO image is slightly patchy (see Fig. 1), and, more importantly, the field of view is not large enough to allow us to properly estimate the sky level. For the above reasons we have decided to adopt the value reported by Zheng et al. (1999) for the mean surface brightness of the stream in the NE loop in the $R$ band-i.e., $27.7 \mathrm{mag} \mathrm{arcsec}^{-2}$ and rely on the BBRO image only to determine the morphology, position, and width of the stream in the sky.

In order to determine the total luminosity of the tidal stream, we first calculate the total area covered by the tidal tails. To do so, we estimate the stream width in two different strips, A and B, located in the bright stream piece detected by Shang et al. (1998; see their Fig. 2). The surface brightness distribution in these two strips is shown in Figure 3. The width of the stream in each of these strips is estimated using a Gaussian function, and the full width at half-maximum (FWHM) of these Gaussian fits is used as a measurement of the stream width. To be conservative, we take the minimum of the FWHM measured in both regions (i.e., $\sim 103^{\prime \prime}$; see Fig. 3) as representative of the width of the stream. At the distance of NGC 5907 the above value corresponds to $\sim 7 \mathrm{kpc}$. Subsequently, we calculate the length of the stream by placing elliptical annuli at the inner and outer sides of each loop. Here we consider that the stream of Figure 2 can be described by the loop L1 formed by the SW-SE-E1-W2 arcs and the half-loop L2 formed by the W3-E2 arcs. The area of each loop is then calculated as $A=\pi q\left(a^{2}-b^{2}\right)$, where $a$ and $b$ are the semimajor axes of the elliptical annuli and are related as $b=a-$ FWHM. The minorto-major axis ratio of the ellipses has an estimated value of $q \simeq$ 0.5 for L1 and L2. We find that for L1 $a=540^{\prime \prime}=36.6 \mathrm{kpc}$, so that $A_{\mathrm{L} 1}=728 \mathrm{kpc}^{2}$, and for L2 $a^{\prime}=27.5 \mathrm{kpc}$, which implies $A_{\mathrm{L} 2}=527 \mathrm{kpc}^{2}$. On doing this, the approximate area subtended by these pieces of the stream is $A=A_{\mathrm{L} 1}+A_{\mathrm{L} 2} / 2 \simeq 990 \mathrm{kpc}^{2}$.

Using this estimate of the area of the SE and E2 loops, the reported mean surface brightness of 27.7 mag $\operatorname{~arcsec}^{-2}$ by Zheng et al. (1999; i.e., $\sim 0.217 L_{\odot} \mathrm{pc}^{-2}$ in the $R$ band) implies a total $R$-band luminosity for the stream of $\sim 2 \times 10^{8} L_{\odot}$. This value is 1 order of magnitude brighter than the luminosity reported for the Sgr dwarf galaxy $\left(\sim 1.9 \times 10^{7}\right.$; Majewski et al. 2003), but it is still consistent with the possibility that this complex structure of debris resulted from the disruption of a single dwarf galaxy during a recent interaction with NGC 5907 (see $\S$ 4.2). We have also roughly estimated the total stellar mass of the stream by using the color $R-I=0.5 \pm 0.3$ given in Zheng et al. (1999). Using the prescription given by Bell \& de Jong (2001), the above color implies a $(M / L)_{R} \sim 1.6_{-1.4}^{+15.0}$ and consequently a total stellar mass of $\sim 3.5 \times 10^{8} M_{\odot}$. This value is very uncertain due to the large error in the stream color. So, the total stellar mass should only be taken as a rough estimate of the stellar mass in the stream within an order of magnitude. Given the large uncertainties of our estimate, it is encouraging that the above stellar mass estimate is similar to the one estimated $\left(\sim 2 \times 10^{8} M_{\odot}\right)$ by Johnston et al. (2001) using dynamical considerations.

Unfortunately, none of the individuals in the color-filtered data set were of sufficient exposure length to reveal hints of the faint surrounding structures with enough signal-to-noise ratio to permit detection or surface brightness measurement. Therefore, it is not possible to constrain stellar population gradients along the stream from this study.

\section{4. $N$-BODY SIMULATIONS}

The clear picture of a repeatedly wrapped tidal stream around NGC 5907 naturally suggests fitting the system with $N$-body models. Typically, the number of free parameters implemented by $N$-body models of satellite accretion in external galaxies is vast. In the case of NGC 5907, although the mass within the luminous radius is well constrained by measurements of its circular velocity curve, no information is available on the flattening of its potential on the scales where the tidal remnants are observed $(\sim 10-50 \mathrm{kpc}$ in projection). In addition, the present position and velocity vectors of the progenitor system are unknown, as are its mass and luminosity profiles.

Currently, the only available information on the stream is its surface brightness and projected geometry with respect to the host. This introduces a new free parameter, which is the projection angle 



FIG. 3.-Gaussian fits to the surface brightness distributions of the stream in two different strips (A and B) illustrated in Fig. 2. The FWHMs of these Gaussian fits are

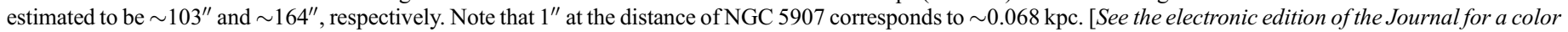
version of this figure.]

between the line of sight and the orbital plane of the stream progenitor. Fortunately, NGC 5907 presents an edge-on disk projection that eliminates a second projection angle between the line-of-sight vector and the axisymmetry plane of the disk. Kinetically, data of bright dynamical tracers (such as planetary nebulae and globular clusters) along the stream would help to disentangle the projection effects and constrain the satellite orbit. Unfortunately, these cannot be readily obtained from individual stars with the present observational capabilities, owing to the large distance of NGC 5907.

The huge degeneracy of the problem weakens any possible constraint obtained through $N$-body modeling of the stream, and, in fact, it is currently unclear how much information can be gleaned from streams in external galaxies using this technique. This is an open question that goes beyond the scope of this paper and will be studied in a future contribution (J. Peñarrubia et al. 2008, in preparation).

Nevertheless, in this paper our $N$-body models are only used to answer a more simple question: can the complex stellar structure be the result of a single merger event? This reduces the problem of finding one of the many possible free parameter combinations that yield a reasonably good match to the observations.

\subsection{Model Parameters}

We examine several illustrative $N$-body simulations of dwarf spheroidal systems disrupting a spiral galaxy with properties similar to NGC 5907. This galaxy is an Sc spiral with a maximum rotational velocity of $V_{c, \max }=227 \mathrm{~km} \mathrm{~s}^{-1}$ and absolute magnitude $M_{B} \simeq-21$ (Casertano 1983) - similar, therefore, to the Milky Way.

Here we assume a static host galaxy potential during the satellite orbit. As Peñarrubia et al. (2006) show, the geometry and kinematics of a tidal stream are independent of the past evolution of the host galaxy potential and solely reflect the present properties of the host. Our satellite galaxy realizations are injected in a host galaxy that has the following components: a Miyamoto \& Nagai (1975) disk, a Hernquist (1990) bulge, and a Navarro et al. (1996, 1997) dark matter halo. The determination of the disk, bulge, and dark matter halo mass from the circular velocity curve of NGC 5907 is very uncertain due to the well-known disk-halo degeneracy. Here we adopt values that are similar to those derived by Just et al. (2006) by fitting the observed rotation curve; the disk and bulge masses are $M_{d}=8.4 \times 10^{10} M_{\odot}$ and $M_{b}=$ $2.3 \times 10^{10} M_{\odot}$, respectively. The radial and vertical exponential scale lengths of the disk are $R_{d}=6.4 \mathrm{kpc}$ and $z_{d}=0.26 \mathrm{kpc}$, respectively. The bulge core radius is $c=0.6 \mathrm{kpc}$. The halo density profile can be written as

$$
\rho_{\mathrm{NFW}}=\frac{\delta_{c} \rho_{c}}{r / r_{s}\left(1+r / r_{s}\right)^{2}},
$$

where $\rho_{c}=277.72 h^{2} M_{\odot} \mathrm{kpc}^{2}$ is the present-day critical density; $h=0.71$ is the Hubble constant in units of $100 \mathrm{~km} \mathrm{~s}^{-1} \mathrm{Mpc}^{-1}$, consistent with constraints from cosmic microwave background (CMB) measurements and galaxy clustering (see Spergel et al. 2007 and references therein); $\delta_{c}$ is a dimensionless parameter; and $r_{s}$ is the halo scale radius. For $\delta_{c}=200$ we use $M_{200}=$ $6.8 \times 10^{11} M_{\odot}, r_{s} \simeq 8.2 \mathrm{kpc}$, and $r_{200}=180 \mathrm{kpc}$. We must remark, however, that different combinations of the host galaxy parameters can yield a similar value of $V_{c, \max }$ (see Just et al. 2006 for a detailed discussion). Unfortunately, this increases the degeneracy inherent to the models of the tidal stream surrounding NGC 5907.

The satellite galaxy is modeled as a King (1966) profile for simplicity. More realistic multicomponent models that distinguish between baryonic and dark matter, such as those motivated by 
$\Lambda$ CDM (e.g., Peñarrubia et al. 2008a, 2008b), would further increase the number of free parameters. Our satellite galaxies have typical parameters for nearby dwarf galaxies (Mateo 1998): a total mass of $2 \times 10^{8} M_{\odot}$, core radius of $R_{c}=390 \mathrm{pc}$, and King concentration $c_{k}=\log _{10}\left(R_{t} / R_{c}\right) \simeq 0.84$, where $R_{t}$ is the King tidal radius. These values were selected to reproduce the projected width and extent of the stellar tidal stream detected in NGC 5907 (see below).

In order to find an illustrative model that provides a reasonable match to the projected distribution of debris, we run a set of $N$-body simulations that explore some of the satellite's orbital and structural parameters. Following Reshetnikov \& Sotnikova (2000), we assume that the orbital apocenter corresponds to the maximum separation of the stream from the center of NGC 5907 (i.e., that of the SE loop), so that $r_{a}=51 \mathrm{kpc}$. To estimate the orbital eccentricity, we vary the pericentric to apocentric distance ratio $\left(r_{p} / r_{a}=0.1,0.2, \ldots, 1.0\right)$. Following the estimates of Johnston et al. (2001), we explore three different progenitor masses $\left(10^{8}\right.$, $2 \times 10^{8}$, and $5 \times 10^{8} M_{\odot}$ ). To derive the age of the stream we integrate each $N$-body realization with a maximum time of $9 \mathrm{Gyr}$, recording the positions and velocities of the $N$-body particles in 70 snapshots (i.e., with a time interval of $0.14 \mathrm{Gyr}$ ). Each snapshot is then visually inspected, varying the projection angle $(\xi)$.

\subsection{Comparison of N-Body Models to the Data}

In Figure 4 we show an illustrative single-accretion model that produces a good overall match to most of the observed structures. This suggests that most of the tidal loops could have formed from the accretion of a single dwarf satellite with an initial total mass of $2 \times 10^{8} M_{\odot}$. For this particular model, the satellite has lost $60 \%$ of its mass due to tidal stripping after $3.6 \mathrm{Gyr}$ of evolution. The progenitor moves on a highly inclined orbit (orbital inclination $i=80^{\circ}$ with respect to the disk plane) with relatively low eccentricity (the pericenter and apocenter are, respectively, $r_{p}=21 \mathrm{kpc}$ and $r_{a}=51 \mathrm{kpc}$, which translates into an intermediate orbital eccentricity of $e \simeq 0.42$ ) and a radial orbital period of $T_{r} \simeq 0.9 \mathrm{Gyr}$. Remarkably, these parameters are fairly similar to those derived for the Sgr stream in the Milky Way (Law et al. 2005). In addition, we find that the projection angle formed by the line of sight and the orbital plane vector has a present value of $\xi \approx 57^{\circ}$ (an edge-on view of the stream implies $\xi=90^{\circ}$ ), which explains the considerable elongation of the stream in the vertical direction.

In general, our model agrees with the previous model of Reshetnikov \& Sotnikova (2000), although a few differences must be noticed. For example, Reshetnikov \& Sotnikova (2000) assume a perfectly polar orbit $\left(i=90^{\circ}\right)$, which is therefore not affected by orbital precession, and derive a slightly higher orbital eccentricity $(\approx 0.6)$, probably a result of the smaller number of observational constraints (only the SE loop was known at that time).

Our model also provides a comprehensive explanation of the relation between the different loops and arcs discussed in $\S 3.1$. In particular, this solution suggests that the leading arm forms the SE loop, bending toward the disk along the E1 loop and giving rise to the W2 arc after it crosses the NGC 5907 disk. The trailing tail of the tidal stream would be visible as the $\mathrm{W} 1$ arc in the west, and its continuation bends toward the east to form the W3 arc and the E2 loop after it crosses the disk. The longest stream piece that fades away toward the west (the SW tail; Fig. 2) corresponds to the oldest piece of the trailing tail with particles that, according to our model, were stripped around 3.6 Gyr ago. Although the shape of our illustrative stream model can reproduce most of the prominent observed features, it also predicts the continuation of the E2 loop that extends between the SE loop and E1 arc. This feature is not obvious in Figure 1, although the presence of an excess of

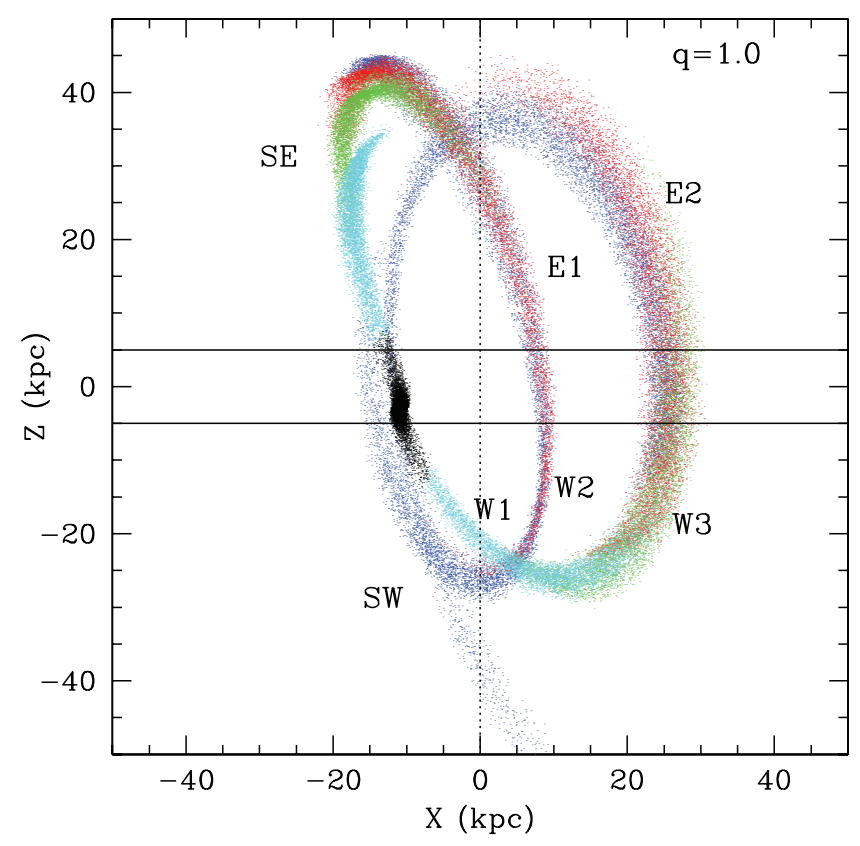

FIG. 4.- Illustrative $N$-body model of the stellar stream detected in NGC 5907. The satellite is realized as a King model with an initial mass, King core, and tidal radii of $M=2 \times 10^{8} M_{\odot}, r_{c}=0.39 \mathrm{kpc}$, and $r_{t}=2.7 \mathrm{kpc}$, respectively (see $\S 4.2$ for details). Different colors denote particles that became unbound after different pericenter passages, whereas black dots indicate particles that remain bound to the main system. Our color code is such that cyan, green, red, and blue indicate particles lost during the first, second, third, and fourth orbital periods, respectively. For this particular model the orbital period is $T_{r}=0.9 \mathrm{Gyr}$. [This figure is also available as an mpeg animation in the electronic edition of the Astrophysical Journal.]

light in the area where this arc would be located (denoted as OV in Fig. 2) is noticeable.

Interestingly, the model also successfully reproduces the strong east-west stream asymmetry (the SE loop reaches a projected distance of $\simeq 51 \mathrm{kpc}$, whereas the SW tail only extends out to $\simeq 27 \mathrm{kpc}$ ), which results from the eccentric orbit of the progenitor system and an inclined line-of-sight projection.

This illustrative model also provides a reasonable explanation for the fate of the progenitor satellite. It suggests that the dwarf could have survived but that its remnant core (Fig. 4, black dots) might currently be hidden behind the galaxy disk, $\sim 15 \mathrm{kpc}$ from the galactic center. If this hypothesis is correct, it leads us to conclude that the NE ring feature of Shang et al. (1998) corresponds to a young piece of the stream. In addition, our model suggests that the NGC 5907 stellar stream may be relatively old, with the fainter, outer loop material (SW tail in Fig. 2) becoming unbound at least 3.6 Gyr ago (Fig. 4, blue dots). The new tidal tails presented in this paper are considerably older than the previous stream pieces discovered by Shang et al. (Reshetnikov \& Sotnikova [2000] estimate the age to be $\lesssim 1.5 \mathrm{Gyr}$ ) and any definitively established piece of the Sgr tidal stream $(\leq 2.5$ Gyr; Majewski et al. 2003; Law et al. 2005). The stellar stream around NGC 5907 may therefore represent one of the most ancient wraps of a tidal stream ever reported in the halo of a spiral galaxy.

\section{DISCUSSION}

In this paper we present in detail an extragalactic perspective of a stellar tidal stream surrounding the nearby spiral galaxy NGC 5907. This ghostly structure is an elegant example of how a single low-mass satellite accretion occurring in the current epoch can produce a very complex, rosette-like structure of debris 
dispersed in the halo of its host galaxy that may survive for several gigayears, a scenario predicted by $N$-body simulations of tidally disrupting stellar systems around the Milky Way (e.g., Law et al. 2005; Peñarrubia et al. 2005; Martin et al. 2005; Martínez-Delgado et al. 2007).

The external view of the stream wrapping NGC 5907 also offers for the first time direct observational evidence that the material stripped from disrupting satellite galaxies (stream stars likely accompanied by dark matter) may cross the inner regions of galactic disks. This process will likely induce gravitational effects (e.g., creation of spiral arms, star formation triggering, etc.) that are not yet fully understood, a phenomenon that has also been suggested for the Sgr stream in our Galaxy (Majewski et al. 2003; MartínezDelgado et al. 2007) but that lacks confirmation due to our inner perspective. In addition, the presence of tidal streams wrapping galaxies with warped disks (see also Martínez-Delgado et al. 2008) may suggest satellite galaxy perturbations as the origin of those features (e.g., Velázquez \& White 1999; Weinberg \& Blitz 2006).

Recent cosmologically motivated simulations have shown that in a $\Lambda C D M$ paradigm the accretion of several massive $\left(0.2 M_{\text {disk }}-\right.$ $0.6 M_{\text {disk }}$ ) subhalos onto the central regions of the host galaxy should be common from $z \simeq 1$ to the present (Kazantzidis et al. 2008). Such accretion events lead to strong warping, flaring, and thickening of an initially cold disk, as well as to the formation of long-lived, ringlike stellar features in the outskirts of the disk that may locate several kiloparsecs off the disk plane and have surface brightnesses in the range of 25-30 mag $\operatorname{arcsec}^{-2}$ (see Fig. 6 of Kazantzidis et al. 2008). Given the similarities in shape and mass between NGC 5907 and the Milky Way, it is interesting to note that our deep images show none of the predicted features besides a classical warped disk. A statistically relevant sample of deep photometric images of nearby, edge-on spiral galaxies might reveal whether the absence of strongly perturbed old disks poses a problem for our theoretical understanding of how disk galaxies form in a $\Lambda$ CDM context.

Our survey reveals that the presence of complex structures from ancient streams in the outskirts of spiral galaxies in the Local Volume ( $\lesssim 15 \mathrm{Mpc}$ ) must be taken into account to correctly interpret the results of deep, pencil-beam photometric probes designed to constrain the formation and composition of galactic halos. This concern is justified in the case of NGC 5907, where several authors (e.g., Sackett et al. 1994; Lequeux et al. 1996) have reported the presence of a peculiar, red, luminous, flattened stellar halo that may actually correspond to tidal debris that in projection appears to be located close below the disk plane and may have originated the faint residual light emission reported in these previous studies (as also suggested by Zheng et al. 1999).

What can we learn from the theoretical study of tidal streams in galaxies beyond the Local Group? First, it must be remarked that theoretical models are severely hampered by the impossibility of measuring the kinematics of individual stars at distances of several Mpc with present instrumentation. In the case of NGC 5907, the large degeneracies in the orbital and structural parameters of the stream progenitor force us to consider our $N$-body model as illustrative only. However, the model is certainly helpful for interpreting most of the observed features of the NGC 5907 tidal stream and proves that such a complex streamlike structure can be explained by the accretion of a single satellite galaxy.

Despite the degeneracy introduced by the observational limitations, an advantage of the panoramic perspective in external galaxies is that it simplifies the detection of stream pieces that were lost several gigayears ago, which strongly increases the number and strength of the constraints that can be derived from these systems. For example, (1) the variation of width and surface bright- ness along tidal streams constrains the number of dark matter subhalos in the Milky Way (Ibata et al. 2002; Johnston et al. 2002; Peñarrubia et al. 2006; Siegal-Gaskins \& Valluri 2008). These authors show that the presence of dark matter subhalos in spiral galaxies would result in progressive heating of tidal streams as a result of close encounters. As Peñarrubia et al. (2006) point out, the average number of dark matter substructures (and, thus, the likelihood of encounters) in a Milky Way-like galaxy has decreased monotonically from $z \sim 2$ to the present, which clearly implies that "old" stream pieces are more likely to reveal perturbations than recently stripped ones. (2) Constraints on the flattening of the host galaxy potential become significantly stronger as the number of stream wraps increases (e.g., Johnston et al. 2001; Law et al. 2005; Peñarrubia et al. 2005). (3) Finally, detecting old stream pieces allows us to study possible metallicity gradients within the progenitor galaxy, as, for example, in the case of the Sgr tidal stream (e.g., Bellazzini et al. 2006; Chou et al. 2007).

The detection of an old, complex tidal stream in NGC 5907 (14 Mpc away) with modest instruments suggests the viability of carrying out extragalactic surveys of substructures in the surroundings of spiral galaxies in the Local Volume. It is a relevant question to wonder how many stellar streams we should expect in the Local Volume. Cosmological $N$-body simulations predict that the incidence of large satellite galaxy accretion occurring in the current epoch is commonly one per spiral galaxy for its inner galactocentric regions, $\lesssim 50 \mathrm{kpc}$ (e.g., Bullock \& Johnston 2005). The presence of such a complex stream at $14 \mathrm{Mpc}$ from us, easily detectable with present observational capabilities, clearly suggests that deep photometric images may reveal a large number of substructures in the stellar halos of spiral galaxies. The different stellar streams detected in the Milky Way (Yanny et al. 2003; Majewski et al. 2003; Jurić et al. 2008; Belokurov et al. 2006a, 2006b) and in M31 (Ibata et al. 2007) by large photometric surveys supports this expectation.

Table 2 gives the confirmed stellar stream features discovered to date. Interestingly, the first outstanding characteristic of the tidal streams discovered in external galaxies to the present day is that there are no detected tidal streams in the Local Group with surface brightnesses as high as seen in NGC 5907.

Currently, observational limitations impede the resolution of stars in external galaxies, which poses a detection threshold of around $\mu_{R} \sim 29$ mag $\operatorname{arcsec}^{-2}$ in unresolved light. In contrast, star counts can reach a detection level as low as $\sim 33 \mathrm{mag} \operatorname{arcsec}^{-2}$ (see, e.g., Belokurov et al. 2006b). This explains why we cannot find the counterparts of the stellar streams detected in the Local Group in external galaxies, but it is indeed puzzling that no tidal stream in the Milky Way or M31 is remotely as bright as the one detected here.

In addition, Table 2 also reveals that, with the single exception of the Sgr dwarf, the progenitor systems of most of the stellar streams known to the present day remain undetected. The most logical explanation is that the progenitor galaxies have been nearly destroyed by now. This scenario is supported by the $N$-body simulations of Peñarrubia et al. (2008b), who show that dwarf galaxies undergoing tidal mass stripping have to lose more than $90 \%-99 \%$ of their initial dark matter halo mass before a stellar tidal stream can start forming. Once the host's tidal field begins to strip stellar material, the central surface brightness $\left(\Sigma_{0}\right)$ and the total luminosity $(L)$ of the dwarf galaxy suffer a sharp, monotonic decline, so that $\Sigma_{0} / \Sigma_{0}(t=0) \propto[L / L(t=0)]^{0.6}$ for $L / L(t=0) \ll 1$. Therefore, as a tidal stream forms, the likelihood of detecting its progenitor decreases until, eventually, the present surface brightness falls beyond the detection limits of our instruments. A second possibility that we explore in our illustrative model (see Fig. 4) 
TABLE 2

Stellar Tidal Streams Detected in Nearby Spiral Galaxies $(D<15 \mathrm{Mpc})$

\begin{tabular}{|c|c|c|c|c|}
\hline Name & $\begin{array}{c}\text { Distance } \\
(\mathrm{Mpc})\end{array}$ & $\mu$ (band) & Progenitor & References \\
\hline \multicolumn{5}{|c|}{ Local Group } \\
\hline 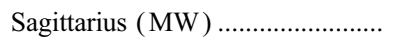 & $\ldots$ & $29.6(\mathrm{~V})$ & Sagittarius dwarf & 1 \\
\hline Monoceros (MW) & $\ldots$ & $34.9(\mathrm{~V})$ & Canis Major dwarf (?) & 2,3 \\
\hline Orphan (MW) & $\ldots$ & $32.4(\mathrm{R})$ & Unknown & 4 \\
\hline M31 giant stream (M31) ................ & 0.78 & $30.0(\mathrm{~V})$ & Unknown & 5,6 \\
\hline \multicolumn{5}{|c|}{ External Galaxies } \\
\hline 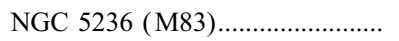 & 5.2 & $26.5(\mathrm{R})$ & Unknown & 7,8 \\
\hline 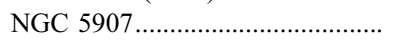 & 11.2 & $26.8(\mathrm{R})$ & Unknown & 9,10 \\
\hline NGC 4013 & 12.0 & $27.0(\mathrm{R})$ & Unknown & 11 \\
\hline NGC 4594 (M104) ....................... & 12.4 & $\ldots$ & Unknown & 8 \\
\hline NGC 3310 ................................. & 14.4 & $25.6(\mathrm{~V})$ & Unknown & 12 \\
\hline
\end{tabular}

REFERENCES.- (1) Martínez-Delgado et al. 2004; (2) Belokurov et al. 2006a; (3) Martin et al. 2005; (4) Belokurov et al. 2007; (5) Ibata et al. 2001a; (6) McConnachie et al. 2003; (7) de Jong et al. 2007; (8) Malin \& Hadley 1997; (9) Shang et al. 1998; (10) this paper; (11) Martínez-Delgado et al. 2008; (12) Whener \& Gallagher 2005.

is that the progenitor systems might be hidden behind the host disk or bulge. Although this scenario may be applied to some of the systems listed in Table 2, it is fairly unlikely that it explains the absence of progenitor galaxies for all of them.

The study of tidal streams in external galaxies is a relatively young field and provides a direct way of addressing some open questions on galactic formation and evolution. For example, studying stellar population gradients along tidal streams via deep Hubble Space Telescope photometry data (see, e.g., Aloisi et al. 2005) will render important constraints on the effect of tides on the stellar formation history of dwarf galaxies. The panoramic view of tidal streams in external galaxies also offers an excellent opportunity to demonstrate tidal stripping of globular clusters formed in satellite galaxies, which may correspond to an important fraction of the globular cluster population of the host, as earlier proposed by Searle \& Zinn (1978). Searching for associated globular clusters and planetary nebulae may also offer a means by which kinematics in distant streams could be derived (i.e., from intrinsically bright absorption-line sources or sources with emission lines), so that kinematical constraints might be placed on theoretical models that aim to reproduce tidal streams in external galaxies. These models, in turn, may provide reasonable estimates of the flattening of the dark matter halos that surround Local Volume galaxies. Ultimately, the ideal scenario would require resolving stellar populations at a distance of $10-20 \mathrm{Mpc}$, which will be feasible in the next one or two decades with large ground-based telescopes like the Thirty Meter Telescope or space-based missions like the James Webb Space Telescope.

We thank H.-W. Rix, E. Bell, A. McConnachie, E. J. Alfaro, R. Ibata, and L. Mayer for very fruitful discussions. We are also grateful to the anonymous referee for constructive suggestions that helped to improve this manuscript. D. M.-D. acknowledges the hospitality of the Max Planck Institute for Astronomy (Heidelberg, Germany), the Institute for Advanced Study (Princeton, New Jersey), and the University of Victoria (Canada), where part of this work was done. D. M.-D. acknowledges funding from the Spanish Ministry of Education and Science (Ramón y Cajal program contract and research project AYA 2007-65090). J. P. thanks Julio F. Navarro for financial support. S. R. M. acknowledges funding by NSF grant AST 03-07851 and NASA/JPL contract 1228235 .
Aloisi, A., van der Marel, R. P., Mack, J., Leitherer, C., Sirianni, M., \& Tosi, M. 2005, ApJ, 631, L45

Barnaby, D., \& Thronson, H. A., Jr. 1992, AJ, 103, 41

Bell, E., \& de Jong, R. S. 2001, ApJ, 550, 212

Bellazzini, M., Newberg, H. J., Correnti, M., Ferraro, F. R., \& Monaco, L. 2006, A\&A, 457, L21

Belokurov, V., Evans, N. W., Irwin, M. J., Hewett, P. C., \& Wilkinson, M. I. 2006a, ApJ, 637, L29

Belokurov, V., et al. 2006b, ApJ, 642, L137 2007, ApJ, 658, 337

Biermann, P. L., \& Munyaneza, F. 2007, preprint (astro-ph/0702173)

Bullock, J. S., \& Johnston, K. V. 2005, ApJ, 635, 931

Casertano, S. 1983, MNRAS, 203, 735

Chou, M.-Y., et al. 2007, ApJ, 670, 346

Davé, R., Spergel, D. N., Steinhardt, P. J., \& Wandelt, B. D. 2001, ApJ, 547, 574

Davies, E. 1990, Machine Vision: Theory, Algorithms, Practicalities (London: Academic)

de Jong, R. S., et al. 2007, in IAU Symp. 241, Stellar Populations as Building Blocks of Galaxies, ed. A. Vazdekis \& R. F. Peletier (Cambridge: Cambridge Univ. Press), 503

\section{EFERENCES}

Dubinsky, J., \& Calberg, R. G. 1991, ApJ, 378, 496

Dumke, M., Braine, J., Krause, M., Zylka, R., Wielebinski, R., \& Guelin, M. 1997, A\&A, 325, 124

Erwin, P., Pohlen, M., \& Beckman, J. E. 2008, AJ, 135, 20

Florido, E., Battaner, E., Gros, A., Prieto, M., \& Mediavilla, E. 1992, Ap\&SS, 190, 293

Forbes, D. A., Beasley, M. A., Bekki, K., Brodie, J. P., \& Strader, J. 2003, Science, 301, 1217

Haralick, R., \& Shapiro, L. 1992, Computer and Robot Vision (Vol. 1; Reading: Addison-Wesley), chap. 7

Hayashi, E., Navarro, J. F., \& Springel, V. 2007, MNRAS, 377, 50

Helmi, A. 2004, ApJ, 610, L97

Hernquist, L. 1990, ApJ, 356, 359

Ibata, R., Irwin, M., Lewis, G., Ferguson, A. M. N., \& Tanvir, N. 2001a, Nature, 412,49

Ibata, R. A., \& Lewis, G. F. 1998, ApJ, 500, 575

Ibata, R. A., Lewis, G. F., Irwin, M. J., \& Quinn, T. 2002, MNRAS, 332, 915 Ibata, R., Lewis, G. F., Irwin, M., Totten, E., \& Quinn, T. 2001b, ApJ, 551, 294 Ibata, R., Martin, N. F., Irwin, M., Chapman, S., Ferguson, A. M. N., Lewis, G. F., \& McConnachie, A. W. 2007, ApJ, 671, 1591

Irwin, J. A., \& Madden, S. C. 2006, A\&A, 445, 123 
James, P. A., \& Casali, M. M. 1998, MNRAS, 301, 280

Johnston, K. V., Choi, P. I., \& Guhathakurta, P. 2002, AJ, 124, 127

Johnston, K. V., Law, D. R., \& Majewski, S. R. 2005, ApJ, 619, 800

Johnston, K. V., Sackett, P. D., \& Bullock, J. S. 2001, ApJ, 557, 137

Johnston, K. V., Spergel, D. N., \& Hernquist, L. 1995, ApJ, 451, 598

Jurić, M., et al. 2008, ApJ, 673, 864

Just, A., Möllenhoff, C., \& Borch, A. 2006, A\&A, 459, 703

Kazantzidis, S., Bullock, J. S., Zentner, A. R., Kravtsov, A. V., \& Moustakas, L. A. 2008, ApJ, 688, 254

Kazantzidis, S., Kravtsov, A. V., Zentner, A. R., Allgood, B., Nagai, D., \& Moore, B. 2004, ApJ, 611, L73

King, I. R. 1966, AJ, 71, 64

Law, D. R., Johnston, K. V., \& Majewski, S. R. 2005, ApJ, 619, 807

Lequeux, J., Combes, F., Dantel-Fort, M., Cuillandre, J.-C., Fort, B., \& Mellier, Y. 1998, A\&A, 334, L9

Lequeux, J., Fort, B., Dantel-Fort, M., Cuillandre, J.-C., \& Mellier, Y. 1996, A\&A, 312, L1

Majewski, S. R., Skrutskie, M., Weinberg, M., \& Ostheimer, J. 2003, ApJ, 599, 1082

Malin, D., \& Hadley, B. 1997, Publ. Astron. Soc. Australia, 14, 52

Martin, N. F., Ibata, R. A., Conn, B. C., Lewis, G. F., Bellazzini, M., \& Irwin, M. J. 2005, MNRAS, 362, 906

Martínez-Delgado, D., Gómez-Flechoso, M. A., Aparicio, A., \& Carrera, R. 2004, ApJ, 601, 242

Martínez-Delgado, D., Peñarrubia, J., Jurić, M., Alfaro, E. J., \& Ivezić, Z. 2007, ApJ, 660,1264

Martínez-Delgado, D., Pohlen, M., Gabany, R. J., Majewski, S. R., Peñarrubia, J., \& Palma, C. 2008, ApJ, submitted

Mateo, M. L. 1998, ARA\&A, 36, 435

McConnachie, A. W., Irwin, M. J., Ibata, R. A., Ferguson, A. M. N., Lewis, G. F., \& Tanvir, N. 2003, MNRAS, 343, 1335

Miyamoto, M., \& Nagai, R. 1975, PASJ, 27, 533

Morrison, H. L., Boroson, T. A., \& Harding, P. 1994, AJ, 108, 1191

Navarro, J., Frenk, C. S., \& White, S. D. M. 1996, ApJ, 462, 563 1997, ApJ, 490, 493
Peñarrubia, J., Benson, A. J., Martínez-Delgado, D., \& Rix, H. W. 2006, ApJ, 645,240

Peñarrubia, J., McConnachie, A. W., \& Navarro, J. F. 2008a, ApJ, 672, 904

Peñarrubia, J., Navarro, J. F., \& McConnachie, A. W. 2008b, ApJ, 673, 226

Peñarrubia, J., et al. 2005, ApJ, 626, 128

Pohlen, M., Martínez-Delgado, D., Majewski, S. R, Palma, C., Prada, F., \& Balcells, M. 2004, in ASP Conf. Ser. 327, Satellites and Tidal Streams, ed. F. Prada, D. Martínez-Delgado, \& T. J. Mahoney (San Francisco: ASP), 288

Pohlen, M., \& Trujillo, I. 2006, A\&A, 454, 759

Rand, R. J. 1996, ApJ, 462, 712

Reshetnikov, V. P., \& Sotnikova, N. Y. 2000, Astron. Lett., 26, 277

Rudy, R. J., Woodward, C. E., Hodge, T., Fairfield, S. W., \& Harker, D. E. 1997, Nature, 387, 159

Sackett, P. D., Morrison, H. L., Harding, P., \& Boroson, T. A. 1994, Nature, 370,441

Sancisi, R. 1976, A\&A, 53, 159

Sasaki, T. 1987, PASJ, 39, 849

Searle, L., \& Zinn, R. 1978, ApJ, 225, 357

Shang, Z., et al. 1998, ApJ, 504, L23

Siegal-Gaskins, J. M., \& Valluri, M. 2008, ApJ, 681, 40

Skrutskie, M. F., Shure, M. A., \& Beckwith, S. 1985, ApJ, 299, 303

Spergel, D. N., et al. 2007, ApJS, 170, 377

van der Kruit, P. C., \& Searle, L. 1981, A\&A, 95, 105

Velázquez, H., \& White, S. D. M. 1999, MNRAS, 304, 254

Wehner, E. H., \& Gallagher, J. S., III. 2005, ApJ, 618 L21

Weinberg, M. D., \& Blitz, L. 2006, ApJ, 641, L33

Xilouris, E. M., Byun, Y. I., Kylafis, N. D., Paleologou, E. V., \& Papamastorakis, J. 1999, A\&A, 344, 868

Yanny, B., et al. 2003, ApJ, 588, 824

Yoshida, H., Springel, V., White, S. D. M., \& Tormen, G. 2000, ApJ, 535, L103

Zepf, S. E., Liu, M. C., Marleau, F. R., Sackett, P. D., \& Graham, J. R. 2000, AJ, 119, 1701

Zheng, Z., et al. 1999, AJ, 117, 2757 